\title{
La protesta juvenil en las Jornadas de octubre-2019 en Ecuador: contexto, motivos y repertorios
}

\section{The youth protest in October 2019: context, motives and repertoires}

\author{
Francisco Puente-Izurieta \\ FLACSO-Ecuador \\ frandapui@hotmail.com \\ https://orcid.org/000-0001-9929-6072
}

\begin{abstract}
Resumen
En este trabajo se analiza de forma exploratoria la dinámica de la protesta protagonizada por las y los jóvenes del 3 al 5 de octubre de 2019 en Quito-Ecuador. Para cumplir con este objetivo se examinan los factores contextuales que permiten comprender la influencia de la estructura de oportunidades políticas, las percepciones y emociones que impulsaron la activación contenciosa, y aspectos relacionales que permiten comprender la configuración y articulación de los repertorios empleados por los manifestantes durante los primeros días de este ciclo de protesta. En el levantamiento de la información se ha empleado un enfoque de corte etnográfico que triangula observación de campo, entrevistas semi-estructuradas y documentos digitales, a partir de los cuales se examinan los mecanismos involucrados en las protestas desarrolladas durante los días que antecedieron al arribo del movimiento indígena a la ciudad capital y a la movilización convocada por representantes de organizaciones de trabajadores, indígenas y estudiantes. Así, se exponen y discuten brevemente los aportes de la teoría de la acción colectiva y de la sociología de las emociones para explorar una forma de conjugarlas en una comprensión de la activación contenciosa juvenil.
\end{abstract}

\section{Palabras clave}

Jóvenes, contexto político, percepciones, emociones, espacios de pertenencia, repertorios de contienda.

Forma sugerida de citar: Puente-Izurieta, F. (2021). La protesta juvenil en las jornadas de octubre-2019 en Ecuador: contexto, motivos y repertorios. Universitas, 34, pp. 215-234. 


\begin{abstract}
In this work, the dynamics of the protest carried out by young people from October 3 to 5, 2019 in QuitoEcuador is explored in an exploratory way. To meet this objective, the contextual factors that allow understanding the influence of the structure of political opportunities, the perceptions and emotions that drove the contentious activation, and relational aspects that allow understanding the configuration and articulation of the repertoires used by the protesters during the first days of this cycle of protest. In collecting the information, an ethnographic approach has been used that triangulates field observation, semi-structured interviews and digital documents, from which the mechanisms involved in the protests developed during the days prior to the arrival of the indigenous movement to the capital city and to the mobilization called by representatives of workers', indigenous and student organizations. Thus, the contributions of the theory of collective action and the sociology of emotions are briefly exposed and discussed to explore a way to combine them in an understanding of contentious youth activation.
\end{abstract}

Keywords

Context, perceptions, emotions, spaces of belonging, repertoires of conflict.

\title{
Introducción
}

En este trabajo se examina la confluencia de factores estructurales, agenciales y relacionales para analizar la dinámica de la protesta juvenil durante las jornadas del 3, 4 y 5 de octubre del 2019 en Quito-Ecuador. Esta propuesta ubica el análisis en las actuaciones, percepciones y emociones de diversos manifestantes a partir de los aportes de la sociología de la acción colectiva y de la sociología de las emociones, para presentar una comprensión situacional y dinámica de los mecanismos con los que operó la protesta juvenil.

Estos mecanismos consisten en una "clase delimitada de eventos que alteran de manera idéntica o muy similar las relaciones entre un conjunto específico de elementos" (McAdam et al., 2001, p. 24). Estos son de tres tipos: a) contextuales (ej. una decisión económica desfavorable por parte de las autoridades), b) cognitivo-valorativos (ej. la modificación en las representaciones y emociones de los manifestantes que definen una situación conflictiva) y c) relacionales (ej. roles de individuos, grupos o redes). 
En ese sentido, la categoría "juvenil" se remite a "experiencias y formas de acción, definidas por un mayor grado de independencia frente a otras instancias y actores sociales y, por lo tanto, se especifica como un proceso general, abierto, plural y multidimensional" (Urbina-Cortés, 2014, p. 822). Para analizar este proceso proponemos integrar niveles estructurales y agenciales que nos permitan comprender la disposición estratégica de los y las jóvenes en los espacios geográficos y continuidades temporales en los que se concentró la protesta.

Si bien la muestra de informantes no es lo suficientemente amplia como para hacer generalizaciones, este trabajo tiene la virtud de obtener la información in situ y al calor de los hechos, exponiendo lo que los actores hacen y dicen en medio de su participación en la protesta. En ese sentido los datos de los registros de campo, de las entrevistas y del material documental, adquieren relevancia y sentido, en la medida en que permiten representar la configuración de un encadenamiento de factores que expone una idea aproximada de la dinámica de la protesta juvenil.

Así, se aplicaron inmersiones etnográficas en los puntos de concentración de los manifestantes, lo que permitió registrar el modo en que estos espacios fueron ocupados y el rol que diversos manifestantes jugaron dentro de ellos. Dentro de estos espacios se aplicaron entrevistas semi-estructuradas a doce manifestantes (seis hombres y seis mujeres), seleccionados por su presencia en: a) los enfrentamientos contra agentes de la policía, b) en los parques de El Ejido y El Arbolito, y c) en las inmediaciones entre la Casa de la Cultura y el Puente del Guambra. Adicionalmente, el análisis de noticias publicadas en medios digitales permite sustentar la cronología de sucesos, referenciar las expresiones del presidente de la República, resaltar los hechos que caracterizaron cada jornada y constatar la veracidad de las descripciones y relatos.

En la primera parte se analiza la influencia de la "estructura de oportunidades políticas" (Gamson \& Meyer, 1999) y "el uso conflictivo del espacio" (Sewell, 2001). Mientras examinamos la estructura de oportunidades políticas a partir de las percepciones subjetivas de los manifestantes (Tilly, 2003) y de aquellas señales emitidas por los actores políticos "que les animan a utilizar los recursos con los que cuentan para movilizarse" (Tarrow, 1999, p. 89), el uso conflictivo del espacio responde a la articulación de diferentes actividades orientadas a sostener la contienda dentro de unza zona geográfica delimitada (Sewell, 2001). 
En la segunda parte se analizan las emociones que impulsaron la actividad contenciosa y su articulación durante las protestas (Eyerman, 2005), destacando aquellos resortes del compromiso con los que se plantean las demandas y justifican las actividades en las que participan las y los manifestantes (Hopkins et al., 2005).

Finalmente, en la tercera parte, el análisis de los repertorios de contien$d a$ no se remite a una lista estable y cerrada de formas de acción repetidas e idénticas, sino a "actuaciones constantemente reinventadas y redefinidas bajo el doble efecto de la improvisación y el intercambio de golpes entre oponentes" (Mathieu, 2012, p. 570), es decir, a las formas diferenciadas y articuladas de acción colectiva, redefinidas por los manifestantes durante esos días (McCarthy, 1999; McAdam, 2010).

Con el fin de superar las oposiciones entre los niveles macro y micro-sociológicos, así como entre las perspectivas objetivista y subjetivista, que consideran las conductas contenciosas como esencialmente racionales o emocionalmente sobre determinadas por el espacio y el tiempo en los que se sitúan (Mathieu, 2012), en este trabajo se integran analíticamente la estructura de oportunidades políticas, las emociones inscritas en la activación de los manifestantes, y los formatos de acción que permiten formular una comprensión situacional y dinámica de la protesta (Aminzade \& McAdam, 2001).

\section{Estructura de amenazas políticas: autoritarismo, violencia policial y silencio mediático}

En esta parte analizaremos las declaraciones con las que el presidente de la República reaccionó a las movilizaciones, el rol de la función ejecutiva y de la función legislativa frente a los acontecimientos, y el modo en que la fuerza pública reprimió las manifestaciones. En la segunda parte examinaremos el rol del espacio público en tanto factor estructurante de los repertorios utilizados por los y las manifestantes.

De acuerdo con el modelo propuesto por Ch. Tilly en su famoso libro From mobilization to revolution (1978), la protesta opera como efecto de la exclusión política de grupos que, al no poder defender sus intereses a través de los canales institucionales de rutina, no encuentran otra alternativa que imponerse a través de la disrupción. 
Así, a partir de las 09h00 del 03 de octubre del 2019, miles de estudiantes y jóvenes integrantes de diversas organizaciones militantes de izquierda, se concentraron en las cercanías al puente del Guambra y de la Casa de la Cultura para rechazar la inconsulta expedición del Decreto Ejecutivo $\mathrm{N}^{\circ}$ 883 con el que el Presidente de la República tomaba la decisión de liberar el precio de los combustibles. ${ }^{1}$

Pese al énfasis del primer mandatario en que no habría un aumento drástico en el precio de los bienes y servicios de primera necesidad, popularmente conocido como "paquetazo", ${ }^{2}$ las y los jóvenes marcharon rumbo a las sedes de la Asamblea Nacional, órgano de la función legislativa, y del Palacio de Gobierno, sede de la función ejecutiva: "El presidente mintió y ahora queremos que las autoridades nos den la cara, estamos exigiendo una explicación, que nos escuchen y que se derogue ese decreto" (Pedro, 26 años). Así, con su presencia en las calles, plazas y parques los y las jóvenes se resistían a la pérdida del trabajo de sus padres, al recorte en el presupuesto de educación, al alza de pasajes y a "todo lo que nos están haciendo" (Daniel, 24 años).

Sin embargo, a las $12 \mathrm{~h} 00$ de ese mismo día, el Presidente de la República trasladaba la sede del Gobierno nacional a la ciudad de Guayaquil y, mientras la función legislativa permanecía cerrada, carrocerías blindadas del ejército y de la policía reforzaron los edificios de gobierno para impedir el avance de las marchas que llegaba por las calles aledañas. Los enfrentamientos se desencadenaron cuando los uniformados empezaron a desalojar a los manifestantes, empleando disparos permanentes de diverso tipo de proyectiles que emitían gas lacrimógeno, esquirlas expansivas y balas de goma: "estos explosivos parecen caseros, me acaban de herir en el brazo con fragmentos de hierro oxidado envuelto en cartón" (Luis, 24 años).

Uno de los lugares de enfrentamientos fue la popularmente conocida Plaza del Teatro, cercana a la sede del ejecutivo, donde jóvenes tapados la boca y nariz con sus chompas y mascarillas, incendiaban llantas y levantaban improvisadas barricadas con el material que encontraban a su paso, como ramas de árbol, tubos y piedras. Los manifestantes atestiguan que durante los enfrentamientos "los policías nos atacaban sin razón y usaban sus

1 Desde su posesión, en reiteradas ocasiones el presidente Lenín Moreno había afirmado que no habría "paquetazo". Se puede acceder a un extracto de estas declaraciones en: https://bit.ly/2YHNmKZ; https://bit.ly/3cKjh5w

2 Para mayor detalle sobre el incremento de los bienes y servicios a partir de la aprobación del Decreto Ejecutivo $\mathrm{N}^{\circ} 883$, ver: https://bit.ly/2O2VUK9 
armas sin medida" (Anthony, 25 años), mientras tendían emboscadas para detener a los y las jóvenes que buscaban refugiarse. ${ }^{3}$

A las 20 h00 de esa noche el Primer Mandatario justificó la actuación de la policía, presentando las protestas "bajo el signo delincuencial del saqueo y de la sublevación" (Ramírez- Gallegos, 2020, p. 24). En cadena nacional anunció la expedición del Decreto Ejecutivo $\mathrm{N}^{\circ} 884$, con el que decretaba el estado de excepción en todo el territorio nacional, obedeciendo a la necesidad de: "eliminar esa costumbre que linda con lo miserable de tratar de imponer criterios con actos vandálicos (...), me refiero a los ladrones y criminales que se han dedicado a desestabilizar" (L. Moreno, 2019). ${ }^{4}$ Estas expresiones y "la forma tan violenta en la que fueron tratados nuestros compañeros" (María José, 21 años), generaron sentimientos de indignación, ira y dolor que las y los informantes identificaron con su malestar (Anexo 1).

Para el día siguiente, viernes 4 de octubre, la cantidad de manifestantes y la violencia de los enfrentamientos con la policía revelaron la incipiente configuración estratégica de diversos repertorios de acción, dispuestos en un rango espacial de $2.6 \mathrm{~km}$, en el que el edificio de la Asamblea Nacional se conecta con la Casa de la Cultura y el puente del Guambra, por medio de los parques popularmente conocidos como el Ejido y el Arbolito, hasta el Palacio presidencial ubicado en el centro histórico de la ciudad.

Los repertorios con los que las y los jóvenes ocuparon este espacio permitieron la difusión de la información en tiempo real, organizar el apoyo logístico y volver a la ofensiva, haciendo de este espacio "un desafío a la autoridad" (Sewell, 2001), tanto por el significado estratégico que adquirió para los actores, como por el rol que jugó en el despliegue de la movilización (Anexo 2).

Durante el 5 de octubre, dentro de este mismo espacio, el procedimiento policial para enfrentar los disturbios comenzó con la difusión de un sonido ensordecedor a través de alto-parlantes que impedían a los manifestantes escucharse entre sí. A continuación, los dejaban avanzar para recibirlos con disparos de diversos proyectiles, gas lacrimógeno, e inmediatamente arremeter con sus carrocerías blindadas, motocicletas y caballos. Los perse-

303 de octubre del 2019: "Jóvenes asfixiados y calles cubiertas de piedras dejan las protestas en el Centro Histórico de Quito". Para mayor detalle sobre los resultados del primer día de protestas ver: https://bit.ly/36IoiaU

4 "Presidente Lenín Moreno decreta estado de excepción y ratifica las medidas económicas". Se puede acceder al pronunciamiento completo en: https://bit.ly/3cBjYOC 
guían y dispersaban hasta arrinconarlos y detenerlos, dejando como resultado a cientos de jóvenes heridos, pisoteados por la caballería policial y uno de ellos perdió un ojo en los enfrentamientos (Anexo 3).

Así, la movilización juvenil operó como reacción a las "estructuras de oportunidad creadas por otros" (Tarrow, 1999, p. 89), como las declaraciones del Primer Mandatario y el bloqueo del procesamiento político por parte de las instituciones interpeladas. Adicionalmente, en las respuestas de los informantes podemos advertir la importancia de la represión policial como la "amenaza" (Chazel, 2003) ante la cual se diversificaron, amplificaron y profundización sus niveles y formatos de contención (Della Porta, 1999; Goldstone \& Tilly, 2001).

\section{Significados de la contienda: defensa de derechos y solidaridad}

En este apartado analizamos los significados de la protesta juvenil a partir de las emociones que impulsaron la activación y despliegue de su movilización. En este examen se destaca la modificación de emociones que van del malestar, frente al que se reivindican derechos, hacia el miedo, frente al que la solidaridad dio paso a los repertorios de contienda que sostuvieron la contienda.

Durante la jornada desarrollada el jueves 3 de octubre los y las jóvenes acudieron a las protestas, según María José (21 años) “para reclamar por sus propios derechos y los de todos". Del mismo modo, Pedro (26 años) justificaba su presencia en la marcha al "centro histórico" "porque su papá se quedó sin trabajo, así que él y sus hermanos ya no podrían seguir estudiando". A partir de estas versiones, podemos resaltar un malestar actual e inminente percibido a nivel personal, pero que también recaía sobre sus "mudos sociales" (Vommaro, 2017).

Para quienes protestaban en las calles aledañas al palacio de gobierno, esta era la única forma de resistencia a la imposición, restricción de derechos y precarización de sus condiciones de vida. Así, lo expone Luis (24 años) mientras camina desde el parque El Ejido hacia la Plaza del Teatro alrededor de las 10h00: "Con este decreto suben el pasaje y luego suben todo, así que no nos queda más que salir a las calles". Así, permite dar cuenta de que su disposición contenciosa respondía a la defensa de sus derechos personales y del bienestar de su comunidad, de los que se tiene más o menos 
consciencia en tanto "se comparten los mismos sufrimientos y los mismos sueños de transformación de la opresión” (Bonvillani, 2010, p. 38).

Sin embargo para las $15 \mathrm{~h} 00$, luego de los enfrentamientos con la policía, algunos manifestantes que se replegaban extenuados, sucios y molestos, revelan el sentido ya no solo de malestar, sino de "ira al ver que nos tratan como criminales, cuando protestamos nosotros los jóvenes ahí sí sacan todo su arsenal" (Pablo, 26 años). Es decir que el nivel de violencia generado por el procedimiento de la fuerza pública, activó sentimientos que impulsaron a los y las jóvenes a reanudar sus acciones el siguiente día, ya no solo con el objetivo de reclamar sus derechos, sino para defenderse y atacar a la fuerza pública.

En efecto, para el viernes 4 de octubre, además de culpar al gobierno, los manifestantes denunciaron la agresión policial y la falta de difusión de los enfrentamientos por parte de los medios de comunicación: "Mira cómo nos agrede la policía, pero Teleamazonas (canal de televisión afín al oficialismo) ahí sí no dice nada, eso es lo que nos indigna" (Dayana, 26 años). Lo que se resalta de este hecho es que, pese a este silencio de los medios públicos y privados, los manifestantes estaban conscientes de los riesgos a los que se exponían: “Tengo miedo, muchos compañeros han sido heridos, pero estar aquí es lo único que nos queda. Quiero decir, sino luchamos no vamos a tener qué comer e igual vamos a morir" (Laura, 24 años). Esta consciencia del riesgo permite dar cuenta de la importancia de los medios de difusión alternativos que lograron posicionar un mensaje, permitiendo a las y los manifestantes conocer lo que enfrentaban y sus consecuencias.

Después de casi doce horas de protestas, alrededor de las 20 h00 de esa segunda jornada, se podía seguir el rastro de pequeños grupos y manifestantes solitarios que se retiraban a sus respectivos hogares. Mientras caminaba junto a cuatro amigas por la Av. 6 de Diciembre rumbo a la Av. Colón, hasta llegar a la primera parada de la Ecovía habilitada hacia el norte (sistema de transporte colectivo masivo), Pauli (23 años) accede a comentarnos sus impresiones de ese segundo día de protestas: "Sentí iras, indignación y dolor al ver cómo nos trató la policía por reclamar nuestros derechos, pero igual mañana tenemos que volver a estar aquí". Así, pone en evidencia que el incremento de la ira y el dolor de los manifestantes (así como el de sus familiares y amigos) operó afianzando el compromiso para la lucha (Yang, 2005).

Para el sábado 5 de octubre, el encuentro entre "gente que en el fondo queremos lo mismo" (Esteban, 24 años), además de justificarse por emociones de ira y miedo, también se configuraba como el efecto de sentimientos de soli- 
daridad. "Es verdad que tenemos miedo, pero apoyar a la gente del barrio y a los compañeros de la U es la única manera de enfrentar lo que está pasando" (Esteban, 24 años). Así, es posible constatar que, si bien el contexto político generó reacciones subjetivas compartidas, también el encuentro comunitario en el espacio público produjo la concurrencia de percepciones y emociones comunes, traducidas en acciones de solidaridad (Maffesoli, 1997).

Esta solidaridad se expresó en la articulación de actividades especializadas, con las que los manifestantes cubrieron las necesidades más urgentes de la protesta:

Cuando se caía alguien la gente le ayudaba y gritaban: ¡médico!, mientras lo cargaban. Cuando veíamos que ya no podían por el gas, íbamos con gasas y agua con vinagre para aliviar la irritación de los ojos, y eso fue lo que nos permitió resistir. (Emilia, 24 años)

Así, para el tercer día, ya no se trataba de defender sus derechos y condiciones de vida, sino de la solidaridad para enfrentar la amenaza de la fuerza pública (Mathieu, 2012).

Esta comprensión de la configuración de la solidaridad como forma de lucha puede concebirse como reacción y resultado de la interacción con las amenazas políticas, es decir, en el intercambio de golpes entre los manifestantes y las autoridades. En ese sentido, el malestar, el miedo y la solidaridad cumplieron paulatinamente un papel decisivo en la configuración y diversificación de la acción colectiva (Anexo 4).

Así, podemos comprender la articulación espacio-temporal de los repertorios con los que las y los jóvenes reivindicaron sus derechos, suplieron el silencio de los grandes medios de comunicación y sobrevivieron a la violencia policial, a partir de la configuración de un "nosotros" sustentado en sentimientos de malestar y miedo, que encontró en la solidaridad la plataforma sobre la cual se levantaron redes especializadas de acción que, como veremos a continuación definieron la dinámica de este ciclo de protesta.

\section{Repertorios de contienda: tirapiedras, primeros auxilios y comunicación digital}

En esta sección analizaremos los factores que permiten comprender la activación, diversificación y articulación de los repertorios con los que las 
y los jóvenes sostuvieron este ciclo de protestas, proyectándose como actor colectivo al calor de la contienda (Eyerman, 2005).

El jueves 3 de octubre a las 09h00 un gran grupo de estudiantes marchó desde el puente del Guambra rumbo a la sede de la función ejecutiva, revelando la importancia de las redes, pero sobre todo "de los grupos de chat de las asociaciones estudiantiles y de los colectivos que tenemos entre compañeros para difundir las convocatorias" (María José, 21 años).

Constatar la existencia de estos grupos nos permite dar cuenta de la relevancia de las relaciones de amistad y militancia, expuesta luego en el ejercicio de roles específicos:

Desde aquí puedes ver que unos están arriba dándose con los chapas, otros llevan baldes con agua para apagar las bombas y otros ayudan a pasar las piedras. Todo el mundo hace algo, aunque sea gritar y aplaudir (María José, 21 años). ${ }^{5}$

Así, para el 4 de octubre ya no eran solo estudiantes los que se manifestaban, también se podía identificar a militantes de organizaciones de izquierda como la Juventud Guevarista y la Juventud Antifascista, así como a moradores de los barrios aledaños al centro histórico, que se enfrentaban con palos, piedras y bombas molotov contra la policía. Algunos manifestantes estaban equipados con cascos, mascarillas y guantes, otros tomaban fotografías y transmitían los acontecimientos en tiempo real, mientras otros repartían agua para inhibir los efectos del gas lacrimógeno y prestaban primeros auxilios. Ese día algunos vencieron el miedo, lo hicieron apoyando en lo que podían, defendiendo su vida y la de sus compañeros: "tuve mucho miedo y pensé que me iba a desmayar, pero me dije itengo que ayudarlos!" (Emilia, 24 años).

En los enfrentamientos de esta jornada, comunicadores profesionales $\mathrm{y}$ aficionados voluntarios recibieron fuertes agresiones, una fotorreportera perdió un ojo por el impacto de una bala de goma y varios camarógrafos fueron detenidos. ${ }^{6}$ Gracias a estos intrépidos comunicadores se pudo conocer de primera mano lo que realmente ocurría en diversos puntos y momen-

5 "Chapa" es una palabra de origen quichua que significa seguridad o vigilancia. Coloquialmente, en el Ecuador es el apelativo usado para nombrar despectivamente a los policías.

6 Las historias de la represión en quito durante el paro, sobre el detalle de heridos y detenidos ver: https://bit.ly/3pSGjeo. Para el 05 de octubre la Confederación de Nacionalidades Indígenas del Ecuador-CONAIE, denunciaba heridos en la Sierra centro y un universitario perdió un ojo en los enfrentamientos contra la policía en el centro de Quito. Se puede ver la noticia completa sobre estos hechos en: https://bit.ly/3jnEkML 
tos de la protesta, "acrecentando la indignación popular al desenmascarar el alineamiento con el discurso oficial por parte de los medios tradicionales" (García \& Soria, 2020, p. 402).

El sábado 5 de octubre los enfrentamientos contra la policía empezaron alrededor de las 09h00 y fueron protagonizados por jóvenes que ya no vestían uniformes de sus institutos educativos y además mantenían roles claramente identificables: "nosotras estuvimos en diversos espacios, algunos compañeros estuvieron en la línea delantera, otros, como yo, intentando registrar con mi cámara todo lo que pueda" (Sofi, 23 años).

La primera línea estaba integrada y era constantemente renovada por jóvenes que tiraban piedras y se armaban de palos para enfrentar la arremetida policial, seguidos por manifestantes que los apoyaban y presionaban, proveyéndoles de arsenal, encendiendo hogueras con llantas y ramas, y gritando consignas contra el gobierno. Entre estos grupos se ubicaban comunicadoras digitales que iban y venía estratégicamente para cubrir los acontecimientos. Finalmente, en la tercera línea, que llegaba a los parques El Ejido y El Arbolito, se podía identificar a estudiantes y profesionales de la salud dispuestos a socorrer asfixiados y heridos.

Así, tejidas por historias de malestar, miedo y solidaridad, estas formas de acción se redibujaban en espacios ocupados "entre la integración y la desintegración, entre lo establecido y lo cuestionado" (King, 2005, p. 152), generando procesos de autogestión colectiva, dentro de los que las y los jóvenes sostuvieron la protesta.

En ese sentido, este despliegue de grupos especializados que tiraban piedras para enfrentar a la policía, difundieron contenidos digitales y prestaron primeros auxilios a sus compañeros, solo puede ser entendido a partir de la "micro-movilización" de redes de información y grupos de acción, en los que interactuaban los manifestantes. Así pudieron dirigir sus competencias personales en forma de líneas de acción que rompieron con la rutina y las reglas habituales desplegadas en el espacio-tiempo en el que se configuró la protesta (McAdam, 2010).

\section{Conclusión}

En este trabajo hemos analizado la convergencia de factores contextuales, personales y relacionales para dar cuenta de la dinámica entre agencia y 
estructura durante los primeros días de este ciclo de protesta. En ese sentido, enfocarnos en las percepciones, emociones y repertorios articulados por los jóvenes durante estas jornadas nos ha permitido aproximarnos a una comprensión acerca de la configuración de mecanismos con los que se despliega la protesta juvenil.

A partir de los registros de campo obtenidos durante esas jornadas en los espacios de concentración de los manifestantes, entrevistas a hombres y mujeres que actuaron en estos espacios y de la revisión de material documental publicado en diversos medios digitales, se ha puesto en evidencia el modo en que se configuraron los mecanismos que permiten comprender la dinámica contenciosa: primero la percepción de la estructura de oportunidades y amenazas del sistema político (Tilly, 2003), las emociones que impulsaron la activación contenciosa (Aminzade \& McAdam, 2001) y los repertorios que expresaron la redefinición del sentido de la protesta durante esas jornadas (McAdam, 2010).

En ese sentido, se ha evidenciado que las decisiones y declaraciones del Primer Mandatario y la inefectividad de las instituciones para el procesamiento político de las demandas, despertaron sentimientos de malestar, mientras que la violencia del procedimiento policial y el silencio de los medios tradicionales, generaron miedo e ira, a partir de los cuales los manifestantes justificaron su movilización durante el segundo. Para el tercer día el afianzamiento de la solidaridad frente a los hechos conocidos y sentimientos generados permitió un despliegue coordinado de diversos repertorios de acción en medio de la contienda.

La presencia de los manifestantes en espacios que permitían su resguardo y su amplia distribución en los momentos de retirada, dio paso a la configuración de líneas de acción con las que las y los jóvenes sostuvieron la contienda durante los primeros tres días de este ciclo de protesta. Así, desde la sociología de la acción colectiva y de sociología de las emociones, hemos podido constatar una conexión entre sentimientos frente a las amenazas del sistema político, con la diversificación y profundización de los repertorios de contienda, es decir, dar cuenta de los mecanismos inscritos en los repertorios con los que operó la actividad contenciosa juvenil.

Al examinar los motivos con los que los manifestantes justifican su presencia en las protestas, podemos dar cuenta de que "los contextos no someten a los sujetos por fuera de sus propias percepciones y decisiones" (Dobry, 1986, p. 79), ni hacen de los factores ideológicos "la clave última de su inte- 
ligibilidad" (Chazel, 2003 p. 140), sino que estas dinámicas son, sobre todo, producto de la acción interdependiente de personas situadas social e históricamente, atrapadas en lógicas específicas, reaccionando frente a sus percepciones sobre las amenazas del sistema político e inspirados por emociones defensivas y ofensivas que los llevan a actuar.

Este proceso permite dar cuenta del modo en que se configura la dinámica de la protesta, a través de repertorios activados por el intercambio de apoyos mutuos, impulsados por la solidaridad inscrita en la reacción ante las amenazas del sistema político, a través de los cuales se pretende la defensa de derechos y de la vida tanto de los propios manifestantes como de sus mundos sociales de pertenencia.

Cabe reconocer que, si bien la información utilizada no es suficiente para demostrar a profundidad la dinámica de cada mecanismo con el que se despliega la protesta, se espera haber expuesto datos que ejemplifiquen y conexiones que evidencien la convergencia de factores entre diferentes niveles analíticos, en una comprensión multidimensional de la activación y despliegue contencioso de las y los jóvenes.

En ese sentido, primero se examinó el desempeño de la estructura de oportunidades políticas, exponiendo el carácter autoritario de las decisiones del gobierno y el bloqueo de las instituciones políticas interpeladas por los manifestantes, la violencia del procedimiento policial, y la concupiscencia de los tradicionales medios de comunicación. Así se da cuenta de la configuración de una estructura de amenazas políticas frente a la que se manifiestan percepciones y formas de acción.

En segundo lugar, se analizaron las emociones inscritas en el involucramiento de los y las jóvenes en los espacios y en los repertorios que sostuvieron la protesta durante esos tres días. En ese sentido, se destaca la solidaridad como respuesta ante el miedo y ante el malestar, generados durante el segundo y primer día. Así, la protesta adquirió el carácter de última ratio para sobrevivir, condensando el significado de la disposición contenciosa juvenil.

Finalmente, se ha puesto en evidencia la convergencia de amenazas y solidaridades en las percepciones y emociones de los manifestantes, no sólo respecto a su mera presencia en el espacio público, sino en la activación y articulación de micro-redes, con las que operó la diversificación de los repertorios empleados por los manifestantes. 
De este modo, se ha presentado una aproximación analítica que conjuga la sociología de la acción colectiva y la sociología de las emociones en una breve comprensión de la dinámica de la protesta, destacando las condiciones de tensión y ajuste, bajo las cuales, las y los jóvenes actuaron para expresar su malestar y enfrentar el miedo generado por la configuración de una estructura de amenazas políticas.

En ese sentido, los repertorios empleados revelaron la diversificación de micro-redes que movilizaron recursos personales y colectivos, que los y las jóvenes fueron capaces de articular en la medida en que ocupaban de forma estratégica el espacio público para resistir. Dentro de este contexto, la acción colectiva fue modulada por emociones generadas y fomentadas a través de redes de interacción que permitieron su masiva movilización y protagonismo sostenido en la contienda.

Se ha presentado un breve análisis que integra el examen de la influencia generada por la estructura de oportunidades políticas en la activación contenciosa, el rol de las emociones con las que se activó y sostuvo la protesta y la diversificación de los repertorios empleados durante esos tres días. Así, se ha expuesto la dinámica de una indómita politización juvenil operada entre movilización y movimiento, entre muchedumbre y organización, con el potencial para actuar de forma irruptora y como comunidad a la vez.

Con base en estas reflexiones, se espera haber contribuido con al menos tres objetivos al campo de estudios sobre la participación política juvenil. Primero, analizando la influencia de la estructura de oportunidades políticas en la dinámica de la protesta. Segundo, ofreciendo una comprensión de la modificación de las emociones inscritas en la activación y dinámica de la movilización juvenil. Y, tercero, reflexionando sobre la articulación de mecanismos que permiten comprender la diversificación de los repertorios implementados para sostener la contienda. 


\section{Anexos}

\section{Anexo 1. Tabla de percepciones y emociones expuestas por informantes ubicados en diferentes puntos durante las tres jornadas de protestas}

\begin{tabular}{|c|c|c|c|c|}
\hline Informante & Día & Lugar & Percepciones & Sentimientos \\
\hline Pedro & 03 & Parque El Ejido & Mentiras del Presidente & $\begin{array}{l}\text { Indignación e } \\
\text { impotencia }\end{array}$ \\
\hline Daniel & 03 & Plaza del Teatro & $\begin{array}{l}\text { Falta de respuesta de las } \\
\text { autoridades }\end{array}$ & $\begin{array}{l}\text { Indignación, ira e } \\
\text { impotencia }\end{array}$ \\
\hline Anthony & 03 & Plaza del Teatro & Agresión policial & $\begin{array}{l}\text { Dolor, ira y } \\
\text { solidaridad }\end{array}$ \\
\hline María José & 03 & Casa de la Cultura & $\begin{array}{l}\text { Mal trato de las } \\
\text { autoridades y agresión } \\
\text { policial }\end{array}$ & $\begin{array}{l}\text { Dolor, indignación y } \\
\text { solidaridad }\end{array}$ \\
\hline Pablo & 04 & Parque El Ejido & $\begin{array}{l}\text { Mal trato policial, } \\
\text { solidaridad }\end{array}$ & Indignación e ira \\
\hline Luis & 04 & Parque El Arbolito & $\begin{array}{l}\text { Descontento frente a } \\
\text { medidas }\end{array}$ & Ira y solidaridad \\
\hline Dayana & 04 & $\begin{array}{l}\text { Puente del } \\
\text { Guambra }\end{array}$ & Silencio de los medios & Indignación \\
\hline Pauli & 04 & Casa de la Cultura & $\begin{array}{l}\text { Descontento frente a } \\
\text { medidas y mal traro } \\
\text { policial }\end{array}$ & $\begin{array}{l}\text { Frustración, miedo y } \\
\text { solidaridad }\end{array}$ \\
\hline Esteban & 05 & Parque El Ejido & $\begin{array}{l}\text { Falta de respuestas de las } \\
\text { autoridades }\end{array}$ & $\begin{array}{l}\text { Ira, miedo y } \\
\text { solidaridad }\end{array}$ \\
\hline Emilia & 05 & Parque El Arbolito & $\begin{array}{l}\text { Falta de respuesta de las } \\
\text { autoridades y violencia } \\
\text { policial }\end{array}$ & Dolor y solidaridad \\
\hline Sofi & 05 & Parque El Arbolito & $\begin{array}{l}\text { Falta de respuesta de las } \\
\text { autoridades }\end{array}$ & Solidaridad \\
\hline
\end{tabular}




\section{Anexo 2. Estudiantes, jóvenes y sus familias ocupando el espacio público de forma estratégica para la contienda}
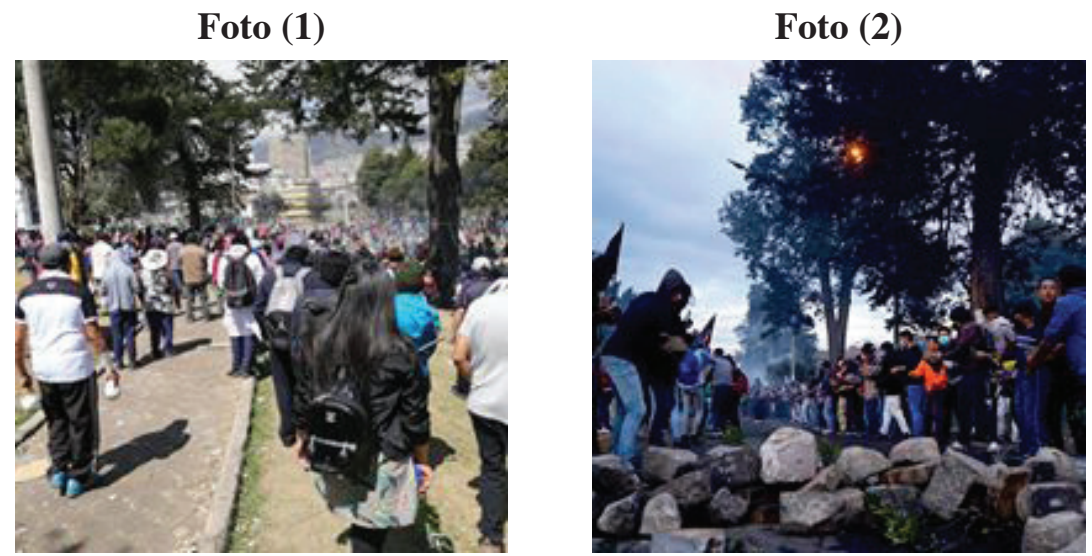

En la foto (1), podemos ver a los y las manifestantes presentes en el Parque El Arbolito. En la foto (2) una fila de jóvenes se organiza para pasar piedras y levantar una barricada en la Av. 6 de Diciembre entre los parque de El Ejido y El Arbolito. Fotos de archivo personal.

\section{Anexo 3}

\section{Fotos (3)}

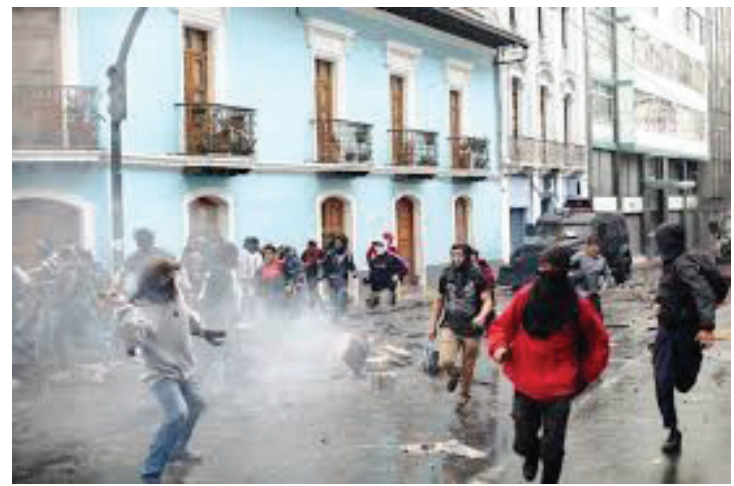


En la foto (3), jóvenes manifestantes huyen de la arremetida policial en las calles Guayaquil y Manabí, a pocas cuadras del palacio presidencial en el "Centro Histórico de Quito". Archivo personal.

\section{Anexo 4. Tabla de repertorios y roles expuestas por los informantes ubicados en diferentes puntos durante las tres jornadas de protestas}

\begin{tabular}{|l|l|l|l|l|}
\hline Informante & \multicolumn{1}{|c|}{ Día } & \multicolumn{1}{c|}{ Lugar } & \multicolumn{1}{c|}{ Repertorio } & \multicolumn{1}{c|}{ Rol } \\
\hline Pedro & 03 & Parque El Ejido & Tirapiedras & Proveer de piedras \\
\hline Daniel & 03 & Plaza del Teatro & Tirapiedras & Sofocar gas lacrimógeno \\
\hline Anthony & 03 & Plaza del Teatro & Comunicación & Tomar Fotos \\
\hline María José & 03 & Casa de la Cultura & Comunicación & Difundir en vivo \\
\hline Pablo & 04 & Parque El Ejido & Tirapiedras & Construir barricadas \\
\hline Luis & 04 & Parque El Arbolito & Tirapiedras & Tirar piedras \\
\hline Pauli & 04 & Casa de la Cultura & Salud & Apoyar en aprovisionamiento \\
\hline Dayana & 04 & Puente del Guambra & Comunicación & Difundir en vivo \\
\hline Esteban & 05 & Parque El Ejido & Salud & Auxiliar heridos \\
\hline Emilia & 05 & Parque El Arbolito & Salud & Auxiliar heridos y asfixiados \\
\hline Sofi & 05 & Parque El Arbolito & Comunicación & Tomar fotos \\
\hline
\end{tabular}

\section{Entrevistas}

- Pedro, 26 años, estudiante de la Universidad Central del Ecuador. Entrevista realizada en el puente del Guambra durante la marcha de los estudiantes hacia el centro histórico de Quito el 03/10/2019 a las 10h00.

- Daniel, 24 años, habitante del barrio San Roque. Entrevista realizada en la concentración de los manifestantes en la Plaza del Teatro el 03/10/2019, a las $15 \mathrm{~h} 00$.

- Anthony, fotógrafo del medio digital Laberinto. Entrevista realizada en la esquina de las calles Esmeraldas y Guayaquil, el 03/10/2019 a las $14 \mathrm{~h} 00$.

- María José, 21 años, militante de la Coordinadora Alternativa de Jóvenes y Estudiantes (CORAJE) y estudiante de la Pontificia Univer- 
sidad Católica del Ecuador. Entrevista realizada en las inmediaciones del Parque El Arbolito el 03/10/2019 a las $11 \mathrm{~h} 00$.

- Luis, 24 años, estudiante de la Pontificia Universidad Católica del Ecuador. Entrevista realizada en la esquina de las inmediaciones del Parque El Ejido el 03/10/2019 a las 12h25.

- Pablo, 26 años, habitante del barrio de La Marín. Entrevista realizada en las inmediaciones del parque el Ejido, el 04/10/2019 a las 12h30.

- Laura, 24 años, habitante del barrio la Tola. Entrevista realizada en las inmediaciones de la Casa del Cultura, el 04/10/2019 a las 15h15.

- Pauli, 23 años, estudiante de la Universidad Politécnica Salesiana. Entrevista realizada en la Av. 6 de Diciembre y Av. Colón (al centro norte de la ciudad) el 04/10/2019 a las 19h00.

- Dayana, 26 años, habitante del barrio el Dorado. Entrevista realizada en las inmediaciones del parque el Arbolito, el 04/10/2019 a las 14h30.

- Esteban, 24 años, estudiante de la Universidad Central. Entrevista realizada en las inmediaciones del Parque El Ejido, el 05/10/2019 a las 11h00.

- Emilia, 24 años, estudiante de la Pontificia Universidad Católica del Ecuador. Entrevista realizada en las inmediaciones de la Casa de la Cultura el 05/10/2019 a las 10h30.

- Sofi, 23 años, estudiante de la Universidad Politécnica Salesiana. Entrevista realizada en las inmediaciones del Parque El Arbolito el 05/10/2019 a las $11 \mathrm{~h} 45$.

\section{Bibliografía}

Aminzade R., \& McAdam D. (2001). Emotions and contentious politics. In R. Aminzade, Jack A. Goldstone, Doug McAdam, Elizabeth J. Perry, William H. Sewell, Sidney Tarrow \& Charles Tilly (Coords.), Silence and Voice in the Study of Contentious Politics (pp. 14-50). Cambridge University Press.

Bonvillani, A. (2017). Pensar en la intemperie. Tensiones ontológicas-epistemológicas y metodológicas en la producción de la "subjetividad política". Quaderns de Psicología, 19(3), 229-240. CiPsi/CIECS-CONICET; Universidad Nacional de Córdoba. https://bit.ly/3tnjL7L

Chazel F. (2003). Du pouvoir à la contestation. LGDJ.

Della Porta, D. (1999). Movimientos sociales y Estado: algunas ideas en torno a la represión policial de la protesta. En: D. McAdam, G. McArthy \& M. 
Zald (Coords.), Movimientos sociales: perspectivas comparadas (pp. 100-142). Istmo.

Eyerman, R. (2005). How social movements move: emotions and social movements. In Helena Flam \& Debra King (Eds.), Emotions and Social Movements (pp. 41-56). Routledge.

Gamson,A., \& Meyer, D. (1999). Marcos interpretativos de la oportunidad política. En D. McAdam, G. McArthy \& M. Zald (Coords.), Movimientos sociales: perspectivas comparadas (pp. 389-412). Istmo.

García, J., \& Soria, E. (2020). Las fracturas de octubre. En Los signos abiertos de octubre contra nuestra América. Estrategias de la derecha en el siglo XXI (pp. 393-410). CLACSO.

Goldstone, J., \& Tilly, Ch. (2001). Threat (and opportunity): popular action and state response in the dynamics of contentious action. In R. Aminzade, Jack A. Goldstone, Doug McAdam, Elizabeth J. Perry, William H. Sewell, Sidney Tarrow \& Charles Tilly (Coords.), Silence and Voice in the Study of Contentious Politics (pp. 179-195). Cambridge University Press.

Hopkins, D., Mckie, L.,Watson, N., \& Hughes, B. (2005). The problem of emotion in care: contested meanings from the Disabled People's Movement and the Feminist Movement. In H. Flam \& D. King (Eds.), Emotions and Social Movements (pp. 119-136). Routledge.

King, D. (2005). Sustaining activism through emotional reflexivity. In H. Flam \& D. King (Eds.), Emotions and Social Movements (pp. 150-169). Routledge.

Maffesoli, M. (1997). Elogio de la razón sensible: una visión intuitiva del mundo contemporáneo. Paidós.

Mathieu, L. (2012). Des mouvements sociaux à la politique contestataire: les voies tâtonnantes d'un renouvellement de perspective. Revue française de sociologie, 3(45), 561-580. https://bit.ly/3aBZbHR

McAdam, D. (2010). Tactical innovation and the pace of insurgency. American Sociological Review, $N^{\circ} 48$, 735-754. https://bit.ly/3tubcry

McAdam, D., Tarrow S., \& Tilly Ch. (2001). Dynamics of contention. Cambridge University Press.

McCarthy, J. (1999). Adoptar, adaptar e inventar límites y oportunidades. En D. McAdam, G. McArthy \& M. Zald (Coords.), Movimientos sociales: perspectivas comparadas (pp. 205-220). Istmo.

Ramírez-Gallegos, F. (2020). Paro pluri-nacional, movilización del cuidado y lucha política. En Octubre y el derecho a la resistencia. Revuelta popular y neoliberalismo autoritario en el Ecuador (pp.11-44). Buenos Aires. 
Sewell, W. (2001). Space in contentious politics. In R. Aminzade, J. Goldstone, D. McAdam, E. Perry, W. Sewell, S. Tarrow, Silence and Voice in the Study of Contentious Politics (pp. 51-88). Cambridge University Press. https:// doi.org/10.1017/CBO9780511815331.003

Tarrow, S. (1999). Estado y oportunidades. La estructuración política de los movimientos sociales. En D. McAdam, G. McArthy \& M. Zald (Coords.), Movimientos sociales: perspectivas comparadas (pp. 71-100). Madrid.

Tilly, Ch. (1978). From mobilization to revolution. Random House.

Tilly, Ch. (2003). The politics of collective violence. Cambridge University Press.

Vommaro, G. (2017). La vida social del mundo político: investigaciones recientes en sociología política, En Gabriel Vommaro \& Mariana Gené (Comps.), Los polvorines. Universidad Nacional de General Sarmiento. 1ra edición.

Yang, G. (2005). Emotional events and the transformation of collective action: the Chinese student movement. In Helena Flam and Debra King (Eds.), Emotions and Social Movements (pp. 79-98). Routledge.

Fecha de envío: 2020/10/23; Fecha de aceptación: 2021/02/04;

Fecha de publicación: 2021/03/01 